\title{
Manejo quirúrgico del angiofibroma nasofaríngeo juvenil
}

\author{
Juan F. Oré ${ }^{1}$, José Saavedra ${ }^{1}$, Ladislao Pasache ${ }^{1}$, Roberto Iwaki ${ }^{1}$, \\ Francisco Avello ${ }^{1}$, Johnny Cárdenas ${ }^{1}$
}

Resumen

Palabras clave

\begin{abstract}
Objetivos: Describir el manejo quirúrgico del angiofibroma nasofaríngeo juvenil, tanto en el abordaje como en la anestesia, evidenciar el grado de compromiso y estadio de los casos intervenidos y determinar las complicaciones y recurrencias del angiofibroma juvenil usando un abordaje Le Fort I. Diseño: Estudio descriptivo retrospectivo. Lugar: Servicio de Cirugía de Cabeza y Cuello, Hospital Dos de Mayo, hospital docente. Participantes: Pacientes con resultado anatomopatológico de angiofibroma juvenil. Intervenciones: Se revisó los casos de intervención quirúrgica de angiofibroma juvenil, confirmado por anatomía patológica entre enero de 1993 hasta diciembre de 2006. Principales medidas de resultados: Resultados quirúrgicos, pérdida sanguínea, complicaciones. Resultados: Se intervino 29 casos en el periodo en estudio, todos varones, con un promedio de edad de 19,2 años y rango de edad entre 13 y 27 años. Procedian principalmente de Lima (34\%) y Cajamarca (17\%). Presentamos 90\% de casos catalogados como Chandler III, 7\% como Chandler IV y 3\% como Chandler II. Todos fueron sometidos a tratamiento quirúrgico, con intubación submentoniana; en 28 pacientes se realizó un abordaje Le Fort I, con osteosíntesis con miniplacas y tornillos de titanio. Se realizó una embolización preoperatorio, al optar por un abordaje transpalatino. El valor promedio de hemoglobina preoperatorio fue 13,6 g\% y en el postoperatorio, $10,5 \mathrm{~g} \%$. El promedio de pérdida sanguínea fue $1019 \mathrm{~mL}$, en un rango de entre 300 y $4500 \mathrm{~mL}$. Se transfundió en promedio 2,3 paquetes globulares por paciente; tres pacientes no requirieron transfusión alguna. No se presentaron complicaciones en el periodo postoperatorio. Conclusiones: El tratamiento quirúrgico es de elección para el tratamiento del angiofibroma juvenil en todos sus estadios. Debido al amplio campo quirúrgico del abordaje Le Fort I y la escasa recurrencia presentada, postulamos este abordaje como el más indicado para todos los estadios de Chandler, en especial para estadios III y IV. La realización del abordaje Le Fort I y la intubación submentoniana engloban diversos conceptos y técnicas que definen su complejidad, como osteotomías del tercio medio facial, técnicas de osteosíntesis, concepto y restauración del plano oclusal del paciente.
\end{abstract}

Angiofibroma; nasofaringe; técnicas quirúrgicas; intubación.

\section{Surgical treatment of juvenile nasopharyngeal angiofibroma}

Abstract

Objectives: To describe the surgical handling of juvenile nasopharyngeal angiofibroma, both approach and anesthesia, to demonstrate compromise and staging and to determine complications and recurrence of juvenile angiofibroma using Le Fort I approach. Design: Retrospective descriptive study. Setting: Head and Neck Surgery Department, Hospital Dos de Mayo. Participants: Patients with pathology confirmed juvenile nasopharyngeal angiofibroma. Interventions: We reviewed all the cases

1 Servicio Cirugía de Cabeza y Cuello, Hospital Dos De Mayo. Lima, Perú. with surgery for juvenile nasopharyngeal angiofibroma confirmed by pathology between January 1993 and December 2006. Main outcome measures: Surgical results, blood loss, complications. Results: We had 29 cases in the study period, all men, with average age 19,2 years, age rank 13 to 27 years. Most cases were from Lima (34\%) and Cajamarca (17\%). We report $90 \%$ of cases catalogued as Chandler III, 7\% as Chandler IV and 3\% as Chandler II. All received surgical treatment with submental intubation; in 28 patients Le Fort I approach was performed with titanium plates and screws osteosynthesis. Preoperative embolization was done when a transpalatine approach was decided. Preoperative hemoglobin was $13,6 \mathrm{~g} \%$ average and postoperative $10,5 \mathrm{~g} \%$. We found an average blood loss of $1019 \mathrm{~mL}$, rank between 300 and $4500 \mathrm{~mL}$. Transfusion 
average was 2,3 red blood-cell packs by patient; three patients did not require transfusion. We did not have any postoperative complication. Conclusions: Surgery is the first treatment choice for any staging of juvenile nasopharyngeal angiofibroma. Due to the large surgical field with Le Fort I approach and few recurrences, we postulate this approach for all Chandler stages, especially Chandler III and IV. The accomplishment of Le Fort I approach and the submental intubation includes diverse concepts and techniques that define their complexity, like middle third facial osteotomies, osteosynthesis techniques, occlusal plane concept and its restoration.

Key words: Angiofibroma; nasopharynx; surgery, operative; intubation.

\section{INTRODUCCIÓN}

El angiofibroma juvenil nasofaríngeo es una patología que se presenta en adolescentes de sexo masculino y entre los 14 y 25 años de edad. Aunque de forma excepcional, se ha descrito casos en mujeres. Representa al 0,5\% de los tumores de cabeza y cuello. Es un tumor benigno, no encapsulado, muy vascularizado, con diseminación submucosa y localmente destructivo $\left({ }^{1-4}\right)$.

Tiene su origen en la nasofaringe, a nivel del agujero esfenopalatino. Además, al crecer ejerce presión sobre las paredes óseas, erosionándolas y llegando a destruirlas. No hace metástasis, pero invade por extensión. Crece con dirección anterior hacia la rinofaringe y la fosa nasal, para comprometer los senos maxilares, seno etmoidal, seno esfenoidal. Lateralmente, se extiende a la fosa pterigomaxilar, alcanzando la fosa infratemporal. A través de esta, invade la cavidad orbitaria por las fisuras orbitarias superiores e inferiores, y la fosa media craneal por los agujeros oval y redondo. Puede así comprometer la silla turca y al seno cavernoso. Es muy raro que destruya la región posterior del seno esfenoidal e infiltre el seno cavernoso, hipófisis o quiasma óptico $\left({ }^{1,2,4}\right)$.

La extensión al seno esfenoidal ocurre en $61 \%$ de los casos y del seno maxilar en $43 \%$. El compromiso intracraneal es más frecuente entre los 13 y 15 años de edad (10\% y $20 \%)\left(^{1,2,5-7}\right)$.

Son tumores de consistencia dura, mamelonados, de color rojizo hasta blanco nacarado. Pueden llegar a tener un gran tamaño, cubiertos con epitelio nasofaríngeo, donde el tumor está constituido por tejido conjuntivo, fibroso y numerosos vasos $\left({ }^{3,6,7}\right)$.

La clínica se presenta con obstrucción nasal progresiva, por lo general unilateral, adicionándose rinolalia, respiración bucal y epistaxis, cada vez más frecuentes y de mayor volumen. Los síntomas se incrementan en relación a la extensión del tumor.

Se suelen agregar signos de invasión y desplazamiento de estructuras, como deformación facial, abombamiento del paladar blando, diplopia, exoftalmos, hipoacusia y cefalea $\left({ }^{1-3,5}\right)$.

Difícil de diagnosticar al inicio, ya que en la rinofaringe no manifiesta síntomas de la lesión primaria. La sospecha es de gran importancia, apoyándose en el interrogatorio y el examen clínico y una rinoscopía anterior y posterior. Ya con el tumor desarrollado, el cuadro sintomático es típico, donde se evidencia un tumor duro, no ulcerado, implantado en las partes altas de la rinofaringe, muy sangrante, en un paciente adolescente masculino $\left({ }^{1-3}\right)$.

La tomografía computarizada es la de mayor utilidad; muestra en cortes axiales y coronales con ventana ósea, las cavidades invadidas por el tumor, así como las destrucción de las paredes óseas. La resonancia es de gran utilidad en los tumores extendidos al endocráneo y a la órbita. La angiografía es importante para demostrar el grado de vascularización y los pedículos nutricios del tumor. Las indicaciones descritas son los casos dudosos, la extensión al seno cavernoso y cuando se decide la embolización preoperatoria $(2,7,8)$.

En el diagnóstico diferencial tenemos a los pólipos antroconales, fibromixoma, craneofaringioma, estesioneuroblastoma, cordoma, rabdomiosarcoma, carcinoma indiferenciado y el linfoma $\left({ }^{1,9}\right)$.

El abordaje quirúrgico y la posibilidad de la combinación de otras terapias, aparte de la cirugía, dependen del estadio tumoral. Existen diferentes clasificaciones, siendo las más usadas la de Fish y Chandler $(1,4,10,11)$ (Tabla 1$)$.

Se describe y realizan diferentes tratamientos, como la hormonoterapia, la quimioterapia -recomendada en lesiones recurrentes y en donde 
Juan F. Oré y col.

Tabla 1. Clasificación de angiofibroma nasofaringeo.

\begin{tabular}{|c|c|c|}
\hline Grado & Clasificación de Fish & Clasificación de Chandler \\
\hline I & $\begin{array}{l}\text { Tumor limitado a la nasofaringe y/o la cavidad } \\
\text { nasal sin invasión ósea. }\end{array}$ & Tumor localizado en la nasofaringe. \\
\hline II & $\begin{array}{l}\text { Tumor que invade la fosa pterigomaxilar o el seno } \\
\text { maxilar o etmoidal o esfenoidal con destrucción ósea. }\end{array}$ & Tumor se extiende a fosa nasal y/o seno esfenoidal. \\
\hline III & $\begin{array}{l}\text { a) Tumor que invade la fosa infratemporal o la } \\
\text { órbita sin invasión endocraneana. } \\
\text { b) Tumor con invasión endocraneana extradural. }\end{array}$ & $\begin{array}{l}\text { Tumor se extiende a uno o más de las siguientes } \\
\text { estructuras: seno maxilar, etmoidal, fosa } \\
\text { pterigomaxilar, fosa infratemporal, órbita y/o mejilla. }\end{array}$ \\
\hline IV & $\begin{array}{l}\text { a) Tumor con invasión endocraneana extradural e } \\
\text { intracraneana sin invasión del nervio óptico, la } \\
\text { silla turca o el seno cavernoso. } \\
\text { b) Tumor con invasión endocraneana extradural e } \\
\text { intracraneana con invasión del nervio óptico, la } \\
\text { silla turca o el seno cavernoso. }\end{array}$ & Tumor que se invade la cavidad craneal. \\
\hline
\end{tabular}

no está indicada la cirugía ni la radioterapia-, la radioterapia, que provoca fibrosis y vasculitis, disminuyendo el tamaño y la vascularización tumoral. Pero, la necesidad de dosis altas junto a la radiación sobre la hipófisis, hipotálamo y nervios ópticos, sumadas a la acción cancerígena de las radiaciones sobre la rinofaringe, limitan sus indicaciones a los tumores de tipo IV, a las recidivas sintomáticas inoperables y en pacientes que rechazan la cirugía. Con la embolización, el objetivo es disminuir el sangrado intraoperatorio, mas debe realizarse no antes de las 96 horas previas a la cirugía, según el material esclerosante utilizado, y además sus indicaciones descritas son los tumores invasores muy vascularizados, en estadios IV, o las recidivas con invasión del seno cavernoso $\left({ }^{1,12-14}\right)$.

La cirugía continúa siendo el método de elección en la actualidad, ya que permite la exploración directa del tumor y de su área de implantación. Además, puede combinarse con la embolización preoperatoria y radioterapia postoperatoria. Existen diversos abordajes utilizados, decidiéndose por un abordaje según la topografía y el volumen del tumor. El abordaje transpalatino es muy usado, pero tiene como desventajas principales el escaso control sobre el pedículo y prolongaciones laterales del tumor, junto a una limitada visión del campo operatorio. En el abordaje transmaxilonasal, se trabaja por medio de una rinotomía lateral o una incisión sublabial, utilizándose o no un microscopio quirúrgico; en el desguante facial o degloving se expone las fosas nasales en su totalidad, necesitando para las prolongaciones en los senos maxilares una antrotomía.

La elaboración de un abordaje Le Fort I implica la realización de una osteotomía de $1 \mathrm{~cm}$. superior al plano descrito para la fractura Le Fort I. Requiere descender en un bloque los maxilares, con apertura del piso nasal, para así obtener amplia visión del tumor y sus prolongaciones, que comprometen los senos maxilares, etmoidales y esfenoidales, llegando a visualizar la base de la fosa craneal media. Requiere de una osteosíntesis con miniplacas y tornillo de titanio, para restaurar la oclusión dental previa del paciente. Debido a la necesidad del uso del bloqueo maxilomandibular, la anestesia suele ser por traqueostomía o submentoniana.

Otros abordajes, como el infratemporal, controlan adecuadamente las prolongaciones en la fosa temporal, pero se suele combinar con otro abordaje; en el abordaje neuroquirúrgico, se aborda el seno cavernoso y la zona selar, estando indicado en tumores estadio IV y en recidivas intracraneanas intradurales $(1,3,4,13-15)$.

El tumor tiende a presentar un comportamiento agresivo, a pesar de ser benigno, por las estructuras comprometidas, como senos paranasales, fosas pterigomaxilar e infratemporal, órbita, e incluso la 
cavidad craneana. Se tiene muy buen pronóstico si se lo reconoce y extirpa temprana e íntegramente. Una variable favorable es la edad del paciente, debido a que después de los 25 años el tumor tiende a regresionar, además que presenta mayor componente fibroso que vascular. Como complicaciones tenemos infecciones, graves hemorragias o secuelas neurológicas por hipoxia cerebral. La malignización es excepcional, tanto sarcomatosa como carcinomatosa $\left({ }^{1,16,17}\right)$.

La remoción meticulosa de la toda la infiltración tumoral es requisito imperativo para evitar las recidivas, con recurrencias encontradas hasta en $40 \%$ de casos. El control postoperatorio tomográfico es necesario para detectar y tratar precozmente las recidivas $(4,18-20)$

El presente artículo nos permite describir la experiencia en cuanto al manejo quirúrgico del angiofibroma juvenil en el hospital, además de establecer el grado de compromiso de las estructuras adyacentes, el estadiaje de los casos intervenidos y determinar las complicaciones y recurrencias, por medio del abordaje Le Fort I, junto a una anestesia submentoniana.

\section{MÉTODOS}

Se realizó un estudio descriptivo retrospectivo, en el que se revisó las historias clínicas y los reportes operatorios de los pacientes sometidos a resección de tumor nasofaríngeo, con resultado anatomopatológico de angiofibroma juvenil, dentro del período comprendido entre enero de 1990 y diciembre de 2006, en el Servicio de Cirugía de Cabeza y Cuello del Hospital Nacional Dos de Mayo.

Se incluyó todos los pacientes intervenidos, con resultado anatomopatológico de angiofibroma juvenil. Se tuvo como criterios de exclusión aquellos pacientes cuyas historias clínicas consignaban datos incompletos o resultados de anatomía patológica no compatible con angiofibroma, teniendo cuatro casos en exclusión.

Se consideró las siguientes variables: edad, departamento de procedencia, antecedentes terapéuticos de importancia, estadiaje según la clasifi- cación de Chandler, operación realizada, volumen de sangrado intraoperatorio (constatado en el reporte operatorio), cantidad y tipo de derivados sanguíneos transfundidos, valores de hemoglobina preoperatorio y control a las 24 horas luego de la cirugía y/o transfusión, complicaciones y resultado del informe anatomopatológico.

La organización, estuvo basada en la identificación de las historias clínicas de las pacientes, por medio de la base de datos en el servicio de admisión, archivo y anatomía patológica del hospital. Se elaboró una hoja de recolección de datos para los resultados obtenidos, siendo procesados con el programa estadístico de Microsoft Excel 2003.

\section{RESULTADOS}

Se identificó 29 casos intervenidos en el periodo descrito, siendo la totalidad de los casos pacientes de sexo masculino.

El rango de edad de los pacientes estuvo entre los 13 y 27 años, con un promedio de 19,2 años y una media de 19 años. El grupo comprendido entre los 16 y 20 años correspondió a 55\% (16/29) de los casos (Figura 1).

En cuanto al lugar de procedencia, $34 \%$ de los casos fue del departamento de Lima, seguido de Cajamarca y Ayacucho, con $17 \%$ y $10 \%$, respectivamente. Cabe destacar que dos casos provinieron de Bolivia, correspondiendo estos a 7\% del total (Figura 2).

En 24 casos (83\%), los pacientes no presentaban antecedentes correspondientes a una intervención terapéutica. Tres casos $(10 \%)$ habían tenido una intervención anterior, por medio de un abordaje transpalatino, en otra institución. Cabe mencionar que en un caso, además del abordaje transpalatino, se realizó un desarme facial unilateral, en el mismo acto quirúrgico.

Además, en solo un caso (3\%), el paciente acudía con un tratamiento radioterápico, con sus dosis completas realizado en otro hospital; dicho paciente presentaba ceguera en el globo ocular del campo irradiado. 


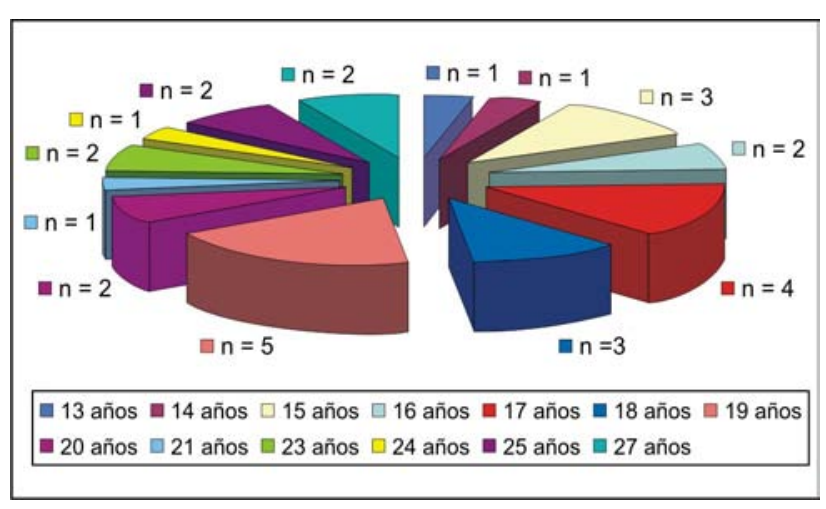

Figura 1. Casos según edades de pacientes.

Solo se realizó en un caso una embolización 72 horas previas al acto quirúrgico, debido a la negativa de los familiares al abordaje Le Fort I; se optó en dicho caso por un abordaje transpalatino.

En el estudio tomográfico solicitado en todos los casos, se evidenció el mayor compromiso de la nasofaringe, en el $100 \%$ de los casos, seguida del cavum, con $97 \%$, y de los senos maxilares, en $89 \%$, unilateral o bilateral (Tabla 2).

Del total de los casos, según la clasificación de Chandler, $90 \%$ (26 casos) fue catalogado como Chandler III, 2 casos (7\%) como Chandler IV y solo hubo un caso de Chandler II (3\%). La totalidad de los pacientes fue sometida a tratamiento quirúr-

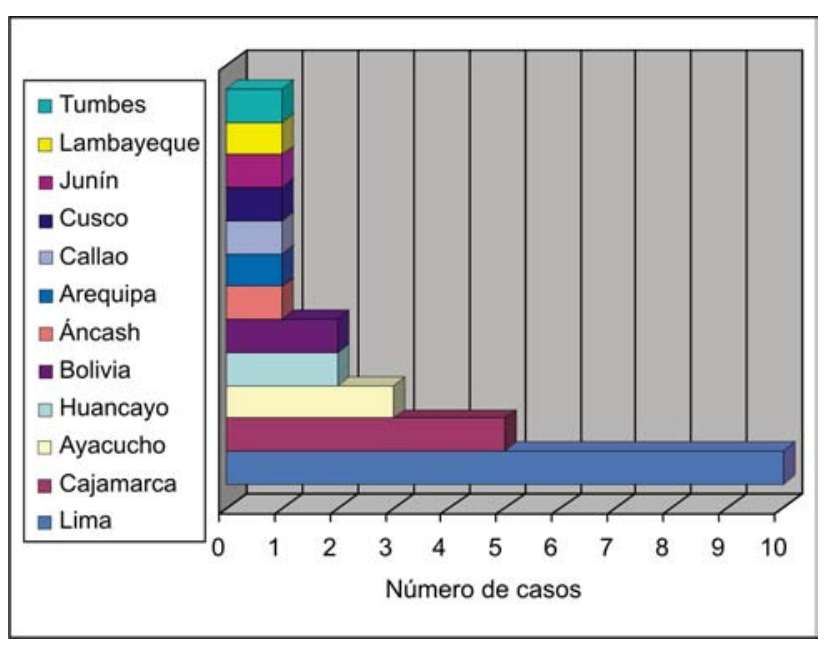

Figura 2. Casos según lugar de procedencia.
Tabla 2. Áreas comprometidas por invasión tumoral.

\begin{tabular}{lcr}
\hline Áreas comprometidas & $\mathrm{N}^{\mathrm{o}}$ casos & $\%$ \\
\hline Nasofaringe & 29 & 100 \\
Cavum & 28 & 97 \\
Seno esfenoidal & 20 & 69 \\
Fosa pterigomaxilar & 16 & 55 \\
Seno maxilar bilateral & 16 & 55 \\
Senos etmoidales & 11 & 38 \\
Seno maxilar unilateral & 10 & 34 \\
Orofaringe & 8 & 28 \\
Seno cavernoso & 2 & 7 \\
Cavidad orbitaria & 1 & 3 \\
Total & 29 & 100 \\
\hline
\end{tabular}

gico, incluyendo los intervenidos con anterioridad, al igual que el sometido a radioterapia.

El $100 \%$ de los pacientes mantenía una vía aérea permeable para la anestesia por medio de una intubación orotraqueal (todos con tubo anillado), con exteriorización submentoniana y fijación a la región cervical, la cual se mantuvo en recuperación, para ser retirado por nuestro servicio, ya con el paciente despierto, a primera hora del día siguiente de la cirugía (Figura 3).

En cuanto al abordaje quirúrgico, en 28 casos (97\%) se realizó una osteotomía Le Fort I, con apertura del piso nasal, exposición de la cavidad nasal, nasofaringe, senos maxilares, senos etmoidales, esfenoidales, llegando a visualizar y palpar la base del piso medio del cráneo (Figura 4).

En solo un paciente se realizó un abordaje transpalatino (3\%). Esto se decidió debido a no haber compromiso de los senos maxilares ni de la cavidad nasal. Se abordó así la nasofaringe, orofaringe, seno esfenoidal y fosa pterigomaxilar, siendo estas áreas las comprometidas por la tumoración.

A todos los pacientes se les realizó la osteosíntesis del trazo de fractura Le Fort I, con miniplacas y tornillos de titanio, previo bloqueo o fijación intermaxilar, restableciendo la oclusión ya sea con tornillos de fijación y alambre quirúrgico 0,16 o con arcos de Erich, alambre 0,16 y ligas dentarias de bloqueo. Igualmente, la totalidad de los pacientes mantuvo un taponamiento nasal anterior y posterior, por un periodo entre 5 y 7 días (Figura 4). 


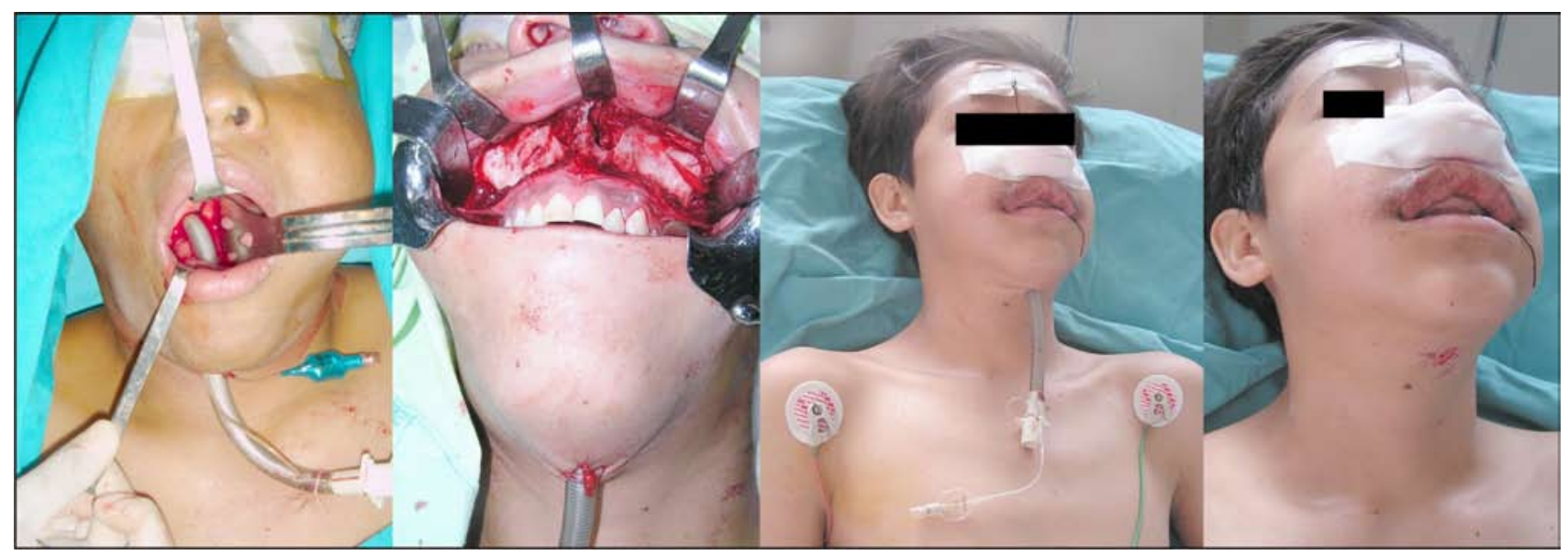

Figura 3. Intubación submentoniana: tubo anillado colocado a través del piso de boca. Nótese el campo operatorio separado del tubo de anestesia. Paciente en recuperación. Ya retirado el tubo, se aprecia la incisión submentoniana.

Se evaluó los valores de hemoglobina preoperatorio y 24 horas luego del término de la transfusión respectiva. Se tuvo como valor promedio de hemoglobina preoperatoria $13,6 \mathrm{~g} \%$ y en el postoperatorio, 10,5 g\%. Cabe resaltar que los valores de hemoglobina prequirúrgicos más encontrados fueron $13 \mathrm{~g} \%$ en 11 casos y $12 \mathrm{~g} \%$ en 6 casos; frente a los valores de control postoperatorio de $11 \mathrm{~g} \%$ en 9 pacientes y $9 \mathrm{~g} \%$ en 7 pacientes (Tabla 3 ).

En cuanto al volumen del sangrado en el acto quirúrgico, se obtuvo un promedio por paciente de $1019 \mathrm{~mL}$. El rango de pérdida sanguínea fue determinado entre 300 y $4500 \mathrm{~mL}$, siendo los valores de pérdida sanguínea más frecuentemente encontrados 600 y $700 \mathrm{~mL}$, en 4 pacientes cada uno (Tabla 4).
Tabla 3. Valores de hemoglobina pre y postoperatorias*.

\begin{tabular}{lcccrr}
\hline $\begin{array}{l}\text { Hemoglobina } \\
\text { preoperatoria }\end{array}$ & $\begin{array}{c}\mathrm{N}^{\mathrm{o}} \\
\text { casos }\end{array}$ & $\%$ & $\begin{array}{c}\text { Hemoglobina } \\
\text { postoperatoria* }\end{array}$ & $\begin{array}{c}\mathrm{N}^{\mathrm{o}} \\
\text { casos }\end{array}$ & $\%$ \\
\hline $10-11 \mathrm{~g} \%$ & 3 & 10 & $7 \mathrm{~g} \%$ & 1 & 3 \\
$12-13 \mathrm{~g} \%$ & 17 & 59 & $8-9 \mathrm{~g} \%$ & 11 & 38 \\
$14-15 \mathrm{~g} \%$ & 6 & 21 & $10-11 \mathrm{~g} \%$ & 15 & 52 \\
$16-17 \mathrm{~g} \%$ & 3 & 10 & $12-13 \mathrm{~g} \%$ & 2 & 7 \\
Total & 29 & 100 & Total & 29 & 100 \\
\hline
\end{tabular}

* Control a las 24 horas del postoperatorio

(pacientes transfundidos y no transfundidos).

Del total de pacientes intervenidos, solo en 3 casos (10\%) no se transfundió algún derivado sanguíneo (paquete globular o plasma fresco congelado), debi-

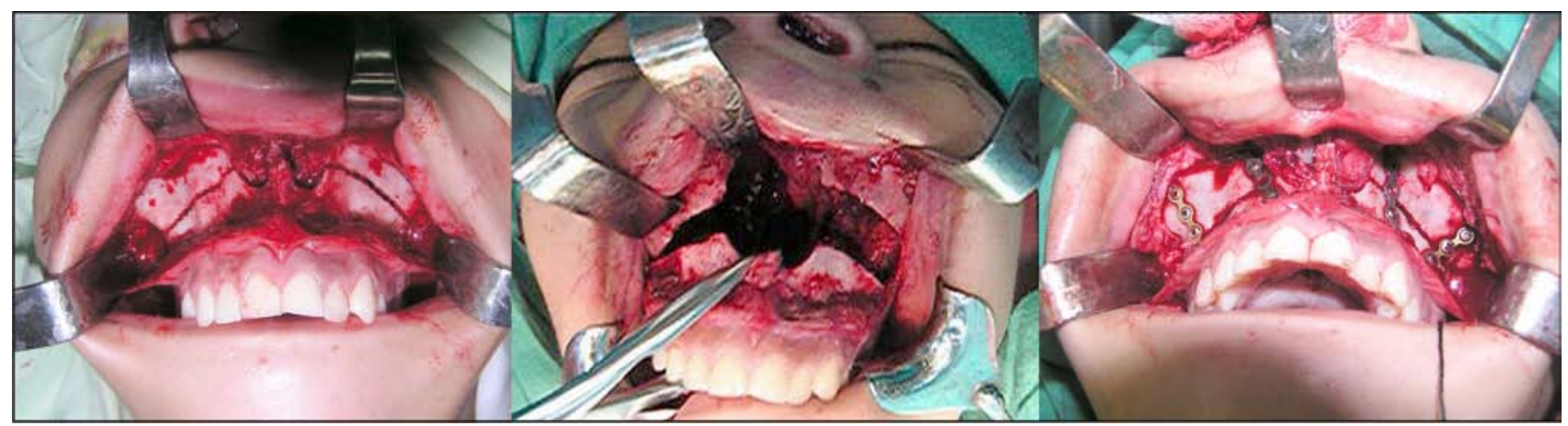

Figura 4. Osteotomía Le Fort I. Vista del campo operatorio con el abordaje descrito. Osteosíntesis con miniplacas y tornillos de titanio (ya retirado el bloqueo intermaxilar). 
Tabla 4. Volumen de sangrado intraoperatorio.

\begin{tabular}{lcc}
\hline Sangrado SOP $(\mathrm{mL})$ & $\mathrm{N}^{\mathrm{o}}$ casos & $\%$ \\
\hline Menos de 500 & 3 & 10 \\
500 a 999 & 17 & 59 \\
1000 a 1499 & 4 & 14 \\
1500 a 1999 & 2 & 7 \\
2000 a 2499 & 2 & 7 \\
Más de 2500 & 1 & 3 \\
Total & 29 & 100 \\
\hline
\end{tabular}

do a que estos pacientes correspondieron a las tres intervenciones con sangrado menor de $500 \mathrm{~mL}$, es decir, 300, 400 y $600 \mathrm{~mL}$; en este último se decidió no transfundir, debido a su valor de hemoglobina preoperatoria de 16,9 g\%. En uno de los casos mencionados, no se transfundió algún derivado sanguíneo, a solicitud del paciente, por motivo de su religión.

Para los 26 pacientes restantes, se transfundió en promedio 2,6 paquetes globulares por caso, siendo la media 2 paquetes globulares. Cabe añadir que en solo 3 casos (10\%) se transfundió en total 4 unidades de plasma fresco congelado, debido a un sangrado mayor a $2000 \mathrm{~mL}$ en la cirugía (Tabla 5).

No se presentaron complicaciones en el periodo postoperatorio, como hemorragia, dificultad respiratoria o mala oclusión dentaria.

En un solo caso, y a los 9 meses posteriores a la cirugía (3\%), se presentó en forma ambulatoria sangrado oral y nasal, lo que nos llevo a pensar en una posible recurrencia de la tumoración.

\section{DISCUSIÓN}

Como menciona la literatura, en nuestra experiencia también todos los pacientes correspondieron a varones jóvenes, en nuestro caso entre 13 y 27 años $(1,3,4,8,14)$.

Podemos observar el predominio de los casos en la población de Lima, posiblemente por ser la capital y la cantidad de inmigración a esta. Evidenciamos también que 16 casos provenían de departamentos de la sierra del Perú y de país altiplánico Bolivia, haciendo referencia a la altura como factor influyente de esta patología.
La frecuencia en tratar a pacientes con estadios Chandler III y IV, en el hospital Dos de Mayo, nos ha hecho optar por un abordaje que nos otorga un amplio campo operatorio, que para muchos cirujanos no es habitual ni el de primera elección. La realización del abordaje Le Fort I y la necesidad de restaurar la oclusión preexistente del paciente por medio de una fijación o bloqueo intermaxilar requiere una vía ventilatoria segura y diferente, para la administración anestésica. Es así que, en la totalidad de los casos se realiza una intubación orotraqueal con salida submentoniana, a través del piso de la boca hasta el triángulo submentoniano. No se presentan complicaciones ni se produce mayor incomodidad al paciente, al optar por esta vía submentoniana de la anestesia, frente a una traqueostomía. Para la osteosíntesis con miniplacas y tornillos de titanio del componente maxilar descendido por la osteotomía Le Fort I, se requiere una fijación intermaxilar realizada siempre luego de la resección tumoral y verificación de la hemostasia del campo operatorio $\left({ }^{3,7,8}\right)$ (Figura 4).

La cantidad de sangrado va de acuerdo a lo expresado en la literatura $(1000 \mathrm{~mL})$, al igual que el requerimiento sanguíneo de 2,3 unidades de sangre por caso (considerando el total 29 de pacientes) $\left({ }^{4,13,15,17}\right)$.

Los mayores sangrados se presentaron en pacientes con estadio IV, radiación y cirugía previa, los cuales fueron factores determinantes que condicionaron una mayor dificultad en la hemos-

Tabla 5. Derivados sanguíneos transfundidos.

\begin{tabular}{lcc}
\hline \multicolumn{1}{c}{ Transfusiones } & $\mathrm{N}^{\mathrm{o}}$ casos & $\%$ \\
\hline Ninguna & 3 & 10 \\
1 Paquete globular & 5 & 17 \\
2 Paquetes globulares & 10 & 34 \\
3 Paquetes globulares & 6 & 21 \\
4 Paquetes globulares & 2 & 7 \\
5 Paquetes globulares + & & \\
1 Unidad de plasma & 2 & 7 \\
7 Paquetes globulares + & & 3 \\
2 Unidades de plasma & 1 & 100 \\
Total & 29 & \\
\hline
\end{tabular}




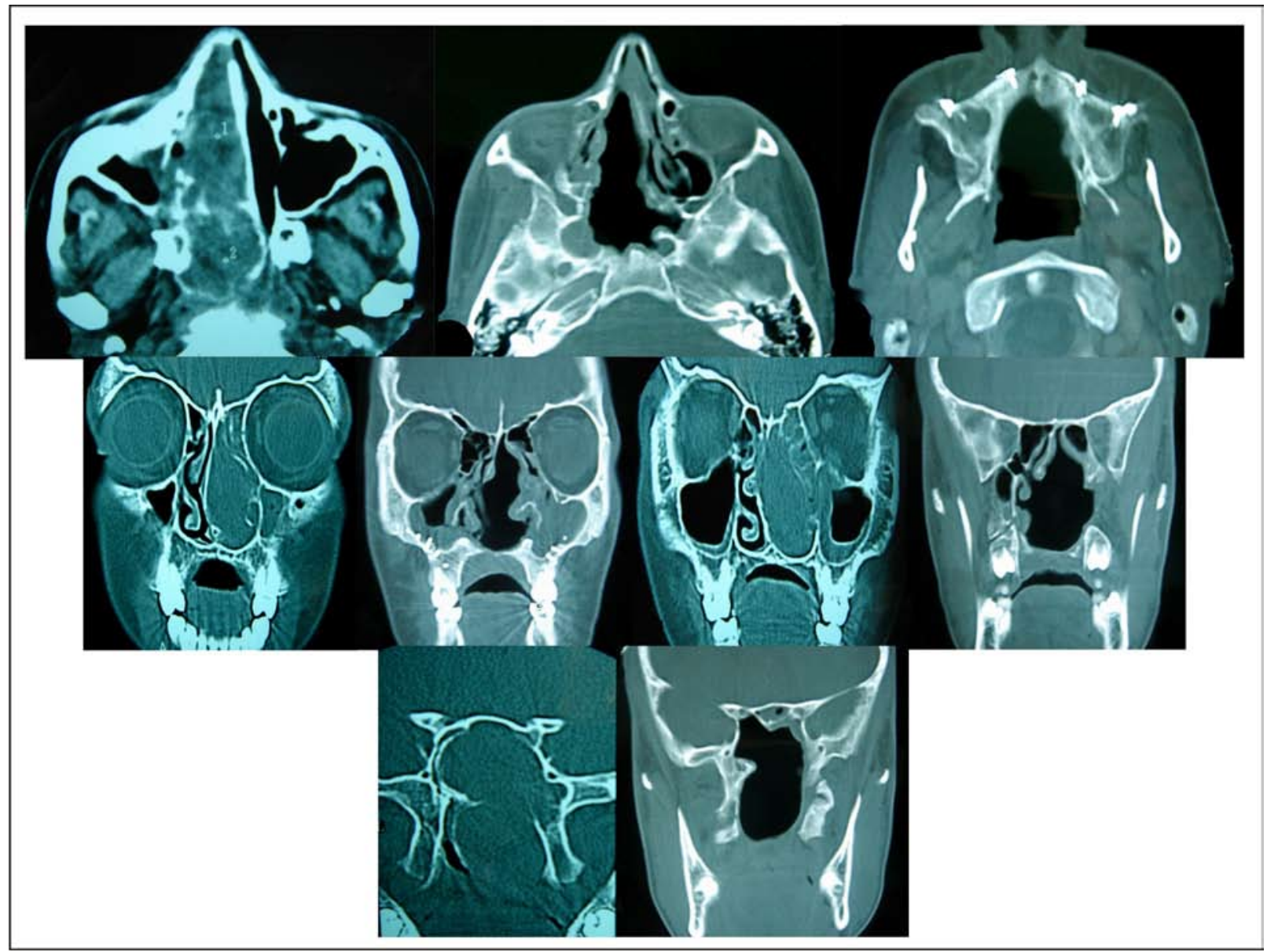

Figura 5. Caso 1. Paciente con angiofibroma nasofaríngeo juvenil estadio Chandler II. Se muestra imágenes tomográficas preoperatorias y postoperatorias en cortes axiales y coronales.

tasia, inmediatamente después de la resección del angiofibroma.

Mas, no encontramos en los pacientes intervenidos con anterioridad mayor diferencia en cuanto a la dificultad de la técnica quirúrgica del abordaje, mas sí al decolaje y exéresis, tanto de la implantación tumoral como de sus prolongaciones. Este hecho debe ser tomado en cuenta, debido a la retracción y amplia fibrosis de los tejidos en los pacientes ya operados; cabe agregar también la friabilidad de los tejidos en caso de la radioterapia.

En nuestros casos, no presentamos complicaciones postoperatorias de la patología en estudio, ni de las propias del abordaje (compromiso nervio infraorbitario, mala oclusión dental, fístulas oro- nasales o sinusales); tampoco presentamos alguna recurrencia tumoral con abordaje Le Fort I $\left({ }^{1,3,4}\right)$ (Figuras 5 y 6).

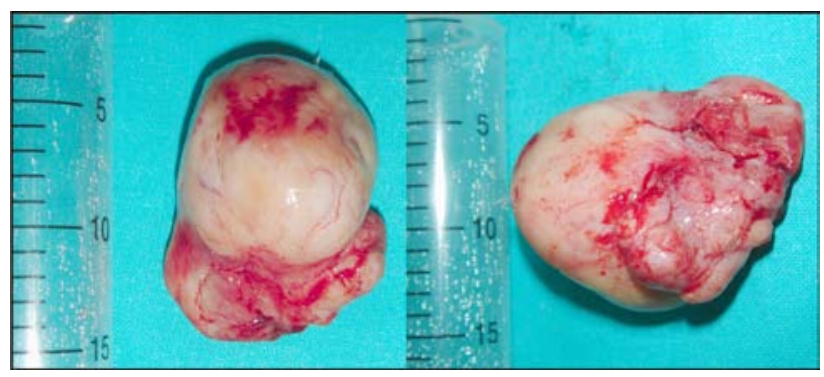

Figura 6. Pieza operatoria del caso descrito, tanto en su cara mucosa como cara de implantación. 


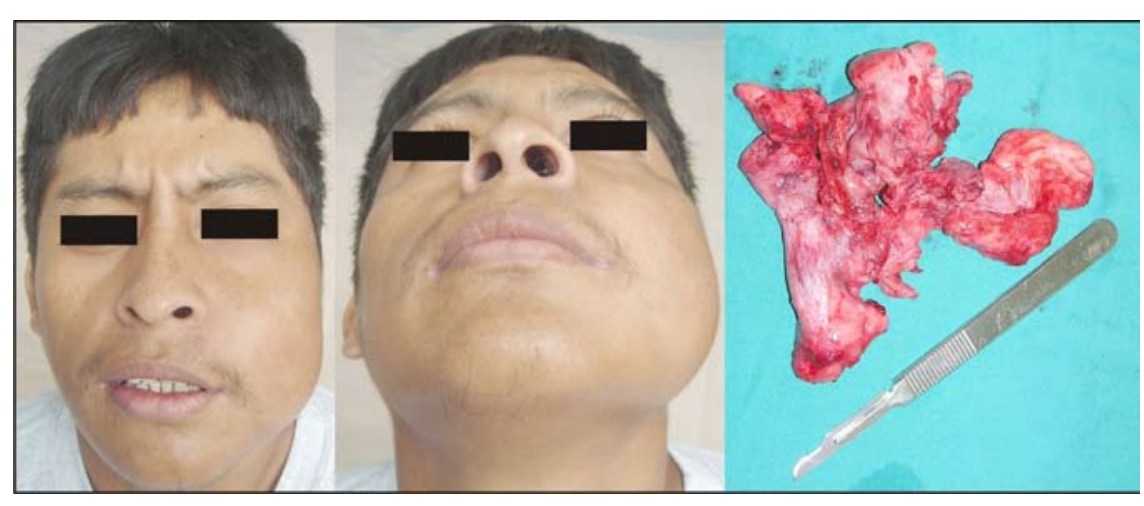

Figura 7. Caso 2. Paciente con angiofibroma nasofaríngeo juvenil estadio Chandler III. Nótese el aumento de volumen de la hemicara izquierda y la asimetría ocular. La pieza operatoria respectiva.

En los cuatro casos excluidos del estudio, catalogados inicialmente como angiofibroma juvenil, tanto por la historia clínica como por el estudio tomográfico, el resultado anatomopatológico evidenció un sarcoma indiferenciado, un estesioneuroblastoma, un pólipo gigante y una displasia fibrosa. Como menciona la literatura, deben ser considerados en el diagnóstico diferencial $(1,6,9,13)$.

El hospital Dos de Mayo, como centro de referencia del Ministerio de Salud, presenta amplia experiencia en el tratamiento de la patología en mención.
Recalcamos que el tratamiento quirúrgico es de elección para el tratamiento del angiofibroma juvenil en todos sus estadíos, siendo lo ideal y de ser posible en estadios tempranos. Es debido a nuestra experiencia en la realización del abordaje Le Fort I, junto al amplio campo quirúrgico y escasa recurrencia tumoral presentada, que postulamos este abordaje como el más indicado para todos los estadios de Chandler, en especial para estadios III y IV (Figuras 7 y 8 ).

Realizar un abordaje Le Fort I implica tener un vasto conocimiento y destreza en las osteotomías del tercio medio facial, la osteosíntesis de la osteotomía en mención, el concepto del plano oclusal del paciente y su perfecta restau-

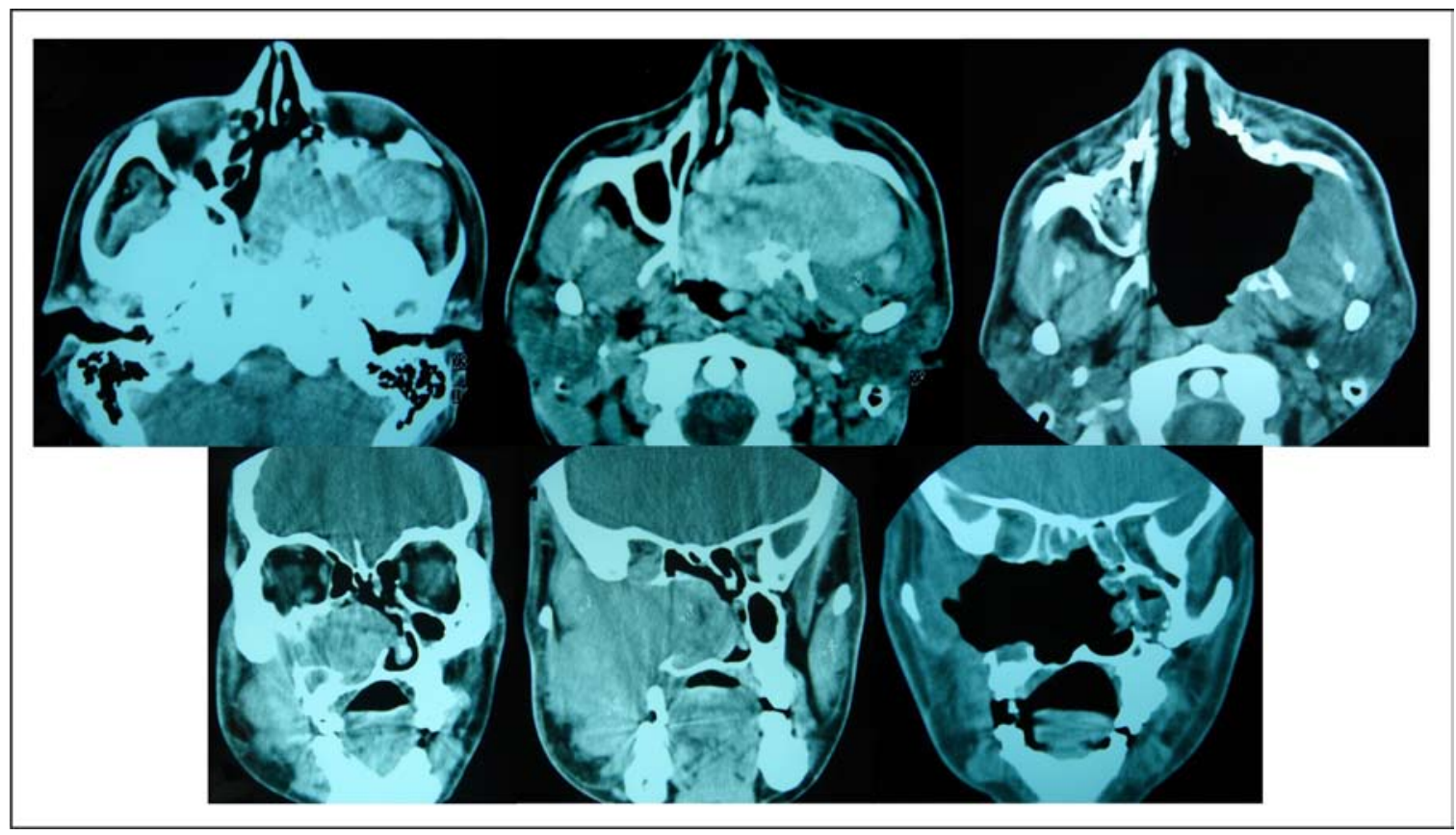

Figura 8. Las respectivas imágenes preoperatorias y postoperatorias del caso anterior, cortes axiales y coronales. Nótese el amplio compromiso y extensión del tumor y las cavidades libres una vez retirado el tumor. 
ración previo el bloqueo maxilar, así como de un control prolijo del campo operatorio, sobre todo en el momento de la resección, junto a la hemostasia. Son todas estas características descritas las que definen la complejidad del abordaje. Sobre la intubación orotraqueal con salida submentoniana, es una técnica segura de realizar y sin dificultad alguna para el retiro del tubo; además, permite el bloqueo maxilar como el trabajo quirúrgico, tanto en la cavidad nasal y oral. Más aún, nos evita la lesión traqueal y sobre todo su potencial complicación tardía, la estenosis traqueal, en caso de decidir por una traqueostomía.

Debido todo lo expuesto anteriormente, se recomienda un entrenamiento adecuado y experiencia del equipo quirúrgico en la realización de este abordaje.

\section{AGRADECIMIENTOS}

Al servicio de Cirugía de Cabeza y Cuello del Hospital Dos de Mayo, por otorgar el apoyo necesario durante la investigación.

\section{REFERENCIAS BIBLIOGRÁFICAS}

1. Chavolla-Magaña R, Labra A, Huerta-Delgado A. Angiofibroma juvenil nasofaríngeo. Metaanálisis de su tratamiento. Rev Med Hosp Gen (Mex). 2003;66(2):71-9.

2. Navarro Vila C, García Marín F, Ochandiano S. Tratado de cirugía oral y maxillofacial. Vol. 3. 1ra Ed. Madrid: Editorial Arán; 2004.

3. Montalván L. Evolución del tratamiento del nasoangiofibroma en el hospital Dos de Mayo, Lima - Perú. Desde la primera vez que se realizó 1978 hasta la fecha 2002 [Tesis segunda especialidad medicina humana]. Lima, Perú: Unidad de Post Grado, Facultad de Medicina Humana, Universidad Nacional Mayor de San Marcos; 2002.

4. Sánchez de Guzmán G. Experiencia en el manejo de nasoangiofibroma juvenil en el Instituto Nacional de Cancerología. Acta Otorrinolaringol Cir Cabeza Cuello (Col). 1999;27(3). Disponible en http://encolombia.com/ otorrino27399contenido.htm

5. Tewfik TL, Tan AK, Al Noury K, Chowdhury K, Tampieri D, et al. Juvenile nasopharyngeal angiofibroma. J Otolaryngol. 1999;28(3):145-51.

6. Gantz B, Seid AB, Weber RS. Nasopharyngeal angiofibroma. Head Neck. 1992;14(1):67-71.
7. Fagan JJ, Snyderman CH, Carrau RL, Janecka IP. Nasopharyngeal angiofibromas: Selecting a surgical approach. Head Neck. 1997;19(5):391-9.

8. Iannetti G, Belli E, De Ponte F, Cicconetti A, Delfín R. The surgical approaches to nasopharyngeal angiofibroma. J Craniomaxilofac Surg. 1994;22(5):311-6.

9. Da Costa DM, Franche GL, Gessinger RP, Strachan D, Nawara G. Surgical experience with juvenile nasopharyngeal angiofibroma. Ann Otolaryngol Chir Cervicofac. 1992;109(5):231-4.

10. Raspall G. Cirugía maxilofacial. Patología quirúrgica de la cara, boca, cabeza y cuello. 1ra Ed. Madrid: Editorial Médica Panamericana; 1997.

11. Ochoa-Carrillo FJ, Carrillo FJ, Frias M. Staging and treatment of nasopharyngeal angiofibroma. Eur Arch Otolaryngol. 1997;254(4):200-4.

12. Gutiérrez-Butanda J, González-Dorantes D, Sosa-de-Martínez C, Mora-Tiscareño M, Medrano-Tinoco M. Angiofibroma nasofaríngeo. Experiencia de diez años en el servicio de otorrinolaringología del Instituto Nacional de Pediatría. Acta Pediatr Mex. 2005;26(3):121-8.

13. Deschler DG, Kaplan MJ, Boles R. Treatment of large juvenile nasopharyngeal angiofibroma. Otolaryngol Head Neck Surg. 1992;106(3):278-84.

14. Avello A. Angiofibroma naso-faringeo juvenil. Rev Cuerpo Méd Hosp Almenara (Per). 1988;12(1):7-9.

15. Posnick J. Craniofacial and maxillofacial surgery in children and young adults. 1ra. Ed. Philadelphia: Editorial Saunders Company; 2000.

16. Loré J, Medina J. Cirugía de cabeza y cuello. 4ta Ed. Buenos Aires: Editorial Médica Panamericana; 2006

17. Shah J, Patel S. Cirugía y oncología de cabeza y cuello. 3ra Ed. Madrid: Editorial Elsevier; 2004.

18. Georgiade G, Riefkohl R, Scout L. Plastic, maxillofacial and reconstructive surgery. 3ra. Ed. Baltimore: Editorial Williams \& Wilkins; 1997.

19. Yoel J. Atlas de cirugía de cabeza y cuello. 1ra Ed. Barcelona: Editorial Salvat; 1986.

20. Herman P, Lot G, Chapot R, Salvan D, Huy PT. Long term follow-up in juvenile nasopharyngeal angiofibromas: analysis of recurrences. Laryngoscope. 1999;109(1):140-7.

Manuscrito recibido el 13 de agosto de 2007 y aceptado para publicación el 25 de setiembre de 2007.

\section{Correspondencia:}

Dr. Juan Francisco Oré Acevedo

Av. Paseo la Castellana 620, dpto. 102, Surco.

Lima 33, Perú

Correo-e:juanfcoore@yahoo.com 